\title{
The Present Situation and Problems of a Dotard Family in Korea
}

\author{
Byung-Chan Min ${ }^{1 *}$, Pill-Kee Min² \\ ${ }^{1}$ Department of Industrial and Management Engineering, Hanbat National University, Korea \\ ${ }^{2}$ Department of Biomedical Engineering, Chonbuk National University, Korea
}

${ }^{*}$ Corresponding author: Department of Industrial and Management Engineering, Hanbat National University, Yusung-gu Daejeon, Korea, E-mail: minbc@hanbat.ac.kr

Received date: January 19, 2017; Accepted date: February 13 2017; Published date: February 20, 2017

Citation: Byung-Chan Min, Pil-Kee Min (2017) Resolution of Tardive Dyskinesia with Clozapine: A Case Report. Dual Diagn Open Acc 1:28.

Copyright: (C) 2017 Min BC, et al. This is an open-access article distributed under the terms of the Creative Commons Attribution License, which permits unrestricted use, distribution, and reproduction in any medium, provided the original author and source are credited.

\section{Understanding of Dementia}

\section{Burden of dementia patients supporters}

The number of dotards over 65 years old based on 2014 in Korea was 610,000 persons, that is, 1 person out of 10 persons. The problem is the family who looks after dementia patients. According to the statistics of health insurance medical expenses in 2013 of Health Insurance Review \& Assessment Service, the medical expenses of dementia patients were $646,200,000,000$ won, which was highest. The National Medical Dementia Center of Korea figures out those medical expenses born by family per 1 dementia patient are $18,920,000$ won. The $62 \%$ of dementia patient's supporters have a mild depressive disorder, and $20 \%$ are receiving psychotherapy. The trouble of the family where a dementia patient exists often leads to a tragedy. As an example, in Dec, 2012, the incident that the grandmother in her seventies attempted to kill her husband who was sleeping at the room by hammering his head several times happened [1]. The grandmother who devotedly had cared for her husband for 5 years confessed, "It was too difficult to live with her dementia husband."

\section{Types of dementia}

Alzheimer type dementia: As a dementia which happens before the age of 65 , it is characterized by that the normal cognitive ability gets damaged besides the severe memory impairment. The only symptom at its early stage is memory impairment, and then impairment happens in memory, judgment, abstract thinking and personality. In the end, at the end of dementia, the patient becomes a vegetative state, the ability of a brain to control physical function is lost, and he or she dies [2]. It is considered that it is impossible to treat the patient.

Multiple cerebral infarction dementias: Unlike Alzheimer Type Dementia, it occurs suddenly and a stroke is frequently experienced over once before dementia emerges. Due to the repetitive stroke, infarction happens on some regions of the brain, which brings about the cognitive impairment. The process of dementia is not constant, so some cognitive functions are affected. However, others are not relatively damaged. At the early stage of dementia, the minor physical symptoms such as headache, dizziness, weakness and fatigue appear, however, the cognitive function gradually declines from this point of time, and when it reaches the final stage, the symptoms similar to Alzheimer Type Dementia appear.

\section{Symptoms and treatment of dementia}

Dementia symptoms: The symptom of cognitive impairment appears.

The phenomena of degradation in memory, learning ability, language function, problem-solving ability, orientation, attention and concentration, judgment, time and space perception ability and social ability occur.

Personality disorder appear as a neuropsychiatric symptom.

The symptoms of depression, aggression, lethargy, indifference, self-centered behavior, anxiety, frustration, delinquency, paranoia, hallucination and delusion, delusional disorder, and insomnia appear.

There is a phenomenon of functional ability impairment.

As the coordination and skills of motor gradually get deteriorated, the physical activities of daily living, the instrumental activities of daily living and protection ability decline [3].

Treatment of dementia: Although many regions which cause dementia get stagnant or develop gradually, many ones may be left dementia status permanently. However, some can be recovered as they are reversible. Of those who suffer from dementia, if they are effectively treated, 35 50\% of them may be completely healed, and their symptoms may be improved. In the end, they may recover the living ability. Recently, the symptoms of dementia can be much improved due to new therapy methods and medication, however, the brain cells which have been already damaged can't be recovered. Therefore, it can be said that there is no perfect treatment on dementia itself yet. Instead, it seems that the continuous management of 
hypertension and stroke, which are the main causes of dementia and the active prevention of dementia, would be important.

The present situation of dotards: It was revealed that the absolute number and incidence rate of dementia get higher with the age increase, and especially that its incidence rate increases rapidly after eighties. In the case of Korea, it is estimated that the number of dotards would be about $313,000,8.3 \%$ of the elderly population of age 65 in 2002, and that as for the level of dementia, 185,000 patients fall under a mild case, 87,000 ones do under an intermediate case, and 41,000 ones do under a severe case (Korea Institute for Health \& Social Affairs, 1997). It is expected that as the average life span of the elderly is likely to increase in the future, the prevalence rate of senile dementia will increase from $8.6 \%$ in 2010 to $9.0 \%$ in 2020 , and that the number of dotards will do from 455,000 to 690,000 . However, as the prevalence rate of senile dementia reported up to now is so varied depending on the researchers, it is considered that the epidemiological investigation of the incidence rate of dementia should be systematically done in the future (Table 1).

Table1: Estimation of Number of Dotards in Korea.

\begin{tabular}{|c|c|c|c|c|c|c|c|}
\hline Division & 1997 & 2002 & 2003 & 2005 & 2010 & 2015 & 2020 \\
\hline Number of elders of 65 and over & 2,929 & 3,772 & 3,969 & 4,366 & 5,302 & 6.345 & 7,667 \\
\hline$(\%)$ & $(6.4)$ & $(7.9)$ & $(8.3)$ & $(9.0)$ & $(10.7)$ & $(12.6)$ & $(15.1)$ \\
\hline Number of Dotards (thousand) & 242 & 313 & 329 & 362 & 455 & 571 & 690 \\
\hline Dementia Prevalence Rate (\%) & 8.3 & 8.3 & 8.3 & 8.3 & 8.6 & 9.0 & 9.0 \\
\hline
\end{tabular}

\section{The Structural Characteristics of Dotard Family}

The dotard family in Korea has some structural characteristics compared to the general elderly family. First, the dotard family is larger in size, compared to the general elderly family. That is, though the number of average household members of general elderly family over 60 years old in Korea gradually shows a tendency to reduce, that of average cohabiting family members of a dotard assumes the aspect that is relatively more [5]. Second, as for family type, in the case of general elderly family, the traditional family type of immediate family is largely maintained, however, the nuclear family type of single, couple family reaches $41 \%$.
The dotard family shows structural characteristics that a proportion of an immediate family is higher than general elderly family, but that of nuclear family is relatively lower. Third, another characteristic of the dotard family is that collateral family where dotards cohabit with the family of their daughters is more, compared with general elderly family. It seems that this aspect comes from the result that dotards want to be cared by their daughters rather than daughters-in-law whom they have no attachment to.

\section{The present situation of support burden of dotards}

Table 2: Plan of Support Burden According to Future Prospect of Dementia.

\begin{tabular}{|l|l|l|l|c|}
\hline Support Plan & Total & Impossibility of Treatment & Aggravation & Maintenance of the status quo / Improvement \\
\hline Family Support & 77.7 & 54.2 & 81.4 & 88.9 \\
\hline Hospitalization & 9.7 & 16.7 & 9.3 & 5.6 \\
\hline Institutional Care & 12.16 & 29.1 & 9.3 & 5.6 \\
\hline Total (\%) & 100 & 100 & 100 & 100 \\
\hline
\end{tabular}

As it is shown in Table 2, though the prospect of dotards is not good, it can be known that the will to support them is high. That is, the case that we will support them within a family was $77.7 \%$, especially, the item of (We'll continue to support them at home where we are supporting them now) showed a high figure. Also, the case that the family wants free or charged elderly facilities showed a lower proportion than the case that they want the protection within a family. Through this, it can be known that the reason that the family wants a support within it accepting the burden is that there still exists repulsion or fear of the facilities or that it thinks that the support is a natural duty of children to parents. Also, it feels the fear of the criticism which might come from a society if it does not support dotards [6].

As a result of examining the subjects whom patients live with, family including sons, daughters, spouses and relatives was highest (62.8\%). Besides that, the subjects who lived alone and those who have entered the facilities accounted for very low proportion, compared with those who lived with family. As a result of examining the subjects who looked after the patients, it was revealed that the family (son, daughter, daughter-in-law, spouse, and relatives) was supporting them (55.8\%). However, 
the proportion that nursing was asked to care-givers and others besides the family was very low (Table 3 ).

Table 3: Analysis of the Subjects who Support the Patients.

\begin{tabular}{|c|c|c|}
\hline \multirow{3}{*}{ Subjects who live with patients } & Family(including children, spouse, and relatives) & $62.8 \%$ \\
\hline & Live Alone & $16.6 \%$ \\
\hline & Others & $20.6 \%$ \\
\hline \multirow{4}{*}{ Subjects who look after patients } & Family(including children, spouse, and relatives) & $55.8 \%$ \\
\hline & Care-givers & $15.9 \%$ \\
\hline & Live Alone & $9.0 \%$ \\
\hline & Others & $19.3 \%$ \\
\hline
\end{tabular}

\section{Support burden of dotard family in Korea}

The $10 \%$ of the elderly population, that is, about 300,000 people are dotards, thus it is estimated that the population who suffers from dementia is about $1,200,000$, the number of the dotards multiplied by their families. In Korea, due to the lack of welfare measures on dementia patients, the main supporters of them are spouses, daughters-in-law, and daughters, which is over $95 \%$. However, while the problem of dementia gets bigger, the capacity that the elderly can be supported within a family is being deteriorated due to the breakup of traditional family structure and the increase in female workforce. In addition, as the social status of women gets improved, the recognition that it is not desirable to solve dementia problem by family unit by forcing the sacrifice of the women who have supported dotards whether voluntarily or reluctantly not is winning significant sympathy. Examining the content of support burden which a dotard family experiences universally, it is as follows:

A limitation on social activities: It is a burden perceived due to the time restriction of the supporters. They consume the time and energy for dotards who have no ability to perform the daily life. If the elders get frustrated and wander as the dementia gets worse. The supporters should enhance the wariness [7]. As a result, they are under much restriction on the contact with social relationship network and social activities, so these wariness and responsibility work as stress to them. However, they come to feel restricted or confined when they can't do what they want to do rather than the fact that they are taking part in the supporting role which they don't want to do.

The negative change in the elderly and family relationship: While the relationship among family members due to dotards remains positive, most of them come to have a negative relationship. As the symptom of dotards gets worse, the supporters gradually feel separated from the life which has been helped and shared. Thus, the relationship between dotards and main supporter's changes from mutual one before the disease to one-sided one, so there would be difficulty in readjustment of relationship according to the role reversal. For example, they show temper or anger to the elders, and they avoid or feel ill at ease with their behaviours.
Conflict in Family Relationship: The difficult situation of supporting dotards leads to the conflict among family members, which aggravates the support burden [8].

The conflict in a family happens in the following cases.

First, it happens when there is no adequate adjustment of the role, time and resources among family members when a dotard happens in their family.

Second, it happens when there is difference in opinion among family members on the judgement of the level of dementia, the attitude towards dotards, and the support strategy.

Psychological Burden: As a result of supporting dotards, there are many cases that the supporters are negatively affected in their psychological wellbeing state. Supporting the dotards, they come to have anxiety and fear on their own image that they get nervous or irritated supporting them and come to quarrel with their family due to that, how to support the elderly and the future of their own and the elderly, which brings about depression. And, with the thought that they haven't treated the elderly well, and that they can't take care of the elderly, they feel guilty and they come to bear the psychological burden on their environmental change due to the change of the elderly.

\section{References}

1. Kim Ki-Tae (2002) The Welfare Practices of the Elderly. Yangseowon Press.

2. Jung-Don K (1995) Korean Dementia Family Study. Hakjisa Press.

3. (1997) Korea Institute for Health \& Social Affairs.

4. Hyung-Keun P (1996) Foreign Dementia Policy and Service Program, A Study on the Elderly Welfare Policy. Research Institute for the Elderly.

5. Son Nam C (2000) Welfare for the Elderly. Hakjisa Press.

6. Heung Sik C (2003) Family Welfare Studies. Hakjisa Press.

7. (2017) Ministry of Health and Welfare in Korea.

8. Alzheimer's Association, Korea.

9. Heung Sik C (2003) Family Welfare Studies. Hakjisa Press. 